\title{
Influence of Local Resistance on Pressure and Speed Changes in Expanded Pneumatic Conveying
}

\author{
Muksin Khodjiev', Ilkhom Abbazov², and Javlon Karimov ${ }^{1}$ \\ ${ }^{1}$ Gulistan State University, Gulistan, Uzbekistan \\ ${ }^{2}$ Jizzakh Polytechnic institute, Jizzakh, Uzbekistan
}

\begin{abstract}
This article focuses on improving product quality and reducing costs to ensure the efficiency of the global production of cotton fiber, in recent years, an increase in the consumption of cotton fiber, its specific type and navigation, changing demand for quality indicators, production of products with certain quality indicators. In all processes of cotton production, as well as in the processes of transporting raw cotton using pneumatic transport, on the basis of analyzes, studies conducted to determine factors that have a negative impact on product quality and their elimination, to create resource-saving technologies that reduce production costs, theoretically the connection is substantiated by the pressure loss of the local resistance of the mixture of air and fibrous waste. Based on the analysis, theoretical equations of motion of a mixture of air and fibrous waste in the expanding part of the pipeline in currently used pneumatic transport systems have been developed. It has been theoretically proven that the angle of expansion of an expanding pipe depends on local resistances generated in the expanding part of the pipe during the movement of air and fibrous waste in the ginner.
\end{abstract}

\section{Introduction}

Large-scale scientific and applied research is being conducted to improve the processes of transporting raw cotton and separating it from the air. In this direction, among other things, the scientific foundations of the process of separating raw cotton from the air that transports it by pneumatic conveyor are being developed and developed, special attention is paid to the creation of scientific automated pneumatic conveying equipment, as well as, through the widespread introduction of resource-saving equipment and technology into production, to improve consumer properties of cotton products. Products made from cotton fiber are the world's leading textile market. For the production of these products, about 25 million tons of cotton fiber are consumed every year, and this figure is growing from year to year [1]. In connection with the increase in consumption of cotton fiber, its certain type and grade, changes in demand for quality indicators, in recent years, special attention is paid to the production of products with certain quality indicators. Accordingly, to ensure the efficiency of cotton fiber production on a global scale, improve product quality and reduce costs, identify and eliminate factors that negatively affect product quality in all processes of cotton production, as well as in the process of transporting cotton by pneumatic transport, create resource-saving technologies that reduce costs production remains one of the most important tasks in this area [2].

In addition to the main processes of the technology of primary processing of raw cotton in the world, large-scale scientific and applied research is being carried out aimed at improving the process of transporting raw cotton through the air and separating it from the air. In this direction, also, the scientific foundations of the process of pneumatic transportation of raw cotton through the air and its separation from the air are being developed and developed, special attention is paid to improving the consumer properties of cotton products by creating scientific automated pneumatic conveying equipment, by accelerating the widespread introduction of modern resource-saving equipment and technologies.

\subsection{The history of the origin of pneumatic transport and its development}

The first pneumatic transport device was used for unloading grain from ships by Dorfman [3] in 1893. The efficiency of the device was small and consumed a lot of energy.

In the cotton ginning industry, the pneumatic conveying device has been used since 1929 [4]. The initial research on the pneumatic transport of raw cotton was introduced by Boris Levkovich. Subsequently, P. Baydyuk made a great contribution to its development. He is the author of experimental studies on the interaction of air with raw cotton in pneumatic transport, the resistance of pneumatic transport to the movement of air and material, movement of material along horizontal and vertical pipes, the radius of action of pneumatic transport, energy consumption during air transportation.

Air transportation of raw cotton contributes to its shrinkage and moisture release from the product. 
T. Makhametov's research on the purification of raw cotton from heavy impurities during pneumatic transportation, A. Ismailov - on increasing the reliability of pneumatic conveying equipment for raw cotton, R. Amirov - on the influence of pneumatic conveying elements on raw cotton and its quality, A. Burkhanov [5] on the interaction of mesh surfaces and bend pipes with the inner surface, research by M. Khasanov in the field of cleaning cotton from fine and heavy mixtures in air transport, research by Kh. Mamarasulov [6] in the field of creating an inertial separator for portable pneumatic conveying equipment, in particular, made a great contribution to the development of the practice and theory of air pneumatic transport. There has been little research on the aerodynamic and local drag of cotton fiber in pipelines [7-9].

\subsection{Research on local resistance}

Local resistances arise when sudden expansion, sharp compression and mixing in separation systems leads to a loss of air pressure as a result of the collision of various obstacles during the joint movement of air and fibrous waste.

There are many types of local resistance, each with a different pressure loss. Therefore, one of the important tasks is to analyze the loss of fiber waste and air flow pressure based on the determination of local resistances in the process of separating fibrous materials from a mixture of air and fiber waste.

Local resistance is the aerodynamic resistance of air ducts assembled in one place.

With local resistances, a restructuring, deviations in the areas of velocities and flow boundaries are always formed. Rebuilding the speed area and maintaining the RPM requires energy consumption. Thus, the passage of air or mixture through the local resistance is always accompanied by a pressure loss. These problems have not been widely studied in terms of theoretical research and analytical development, and the reason for this is that a general analytical method for calculating local resistances has not yet been developed. Along with this, in the cotton ginning industry, the transportation of raw cotton, the aerodynamics of the dusty air purification system have practically not been studied and investigated. There are practically no theoretical foundations in the technological processes of transportation, cleaning and processing of raw cotton in general. Some theoretical researchers such as A. Tadjiboev, B. Mardonov and others conducted research in this direction [10]. However, these studies did not do any research on the separation of fibrous waste from air.

\section{Theoretical studies of a mixture of air and fibrous waste in an expansion pipe}

\subsection{Selection of the flow area for calculating the local resistance in the expanding pipe}

Let us consider an air duct in which the cross-section of the angle of deflection $0<\alpha<\frac{1}{2}\left(0<\alpha \pi<\frac{\pi}{2}\right)$ expands in the direction of the flow of a mixture of air and fibrous waste (Fig. 1).

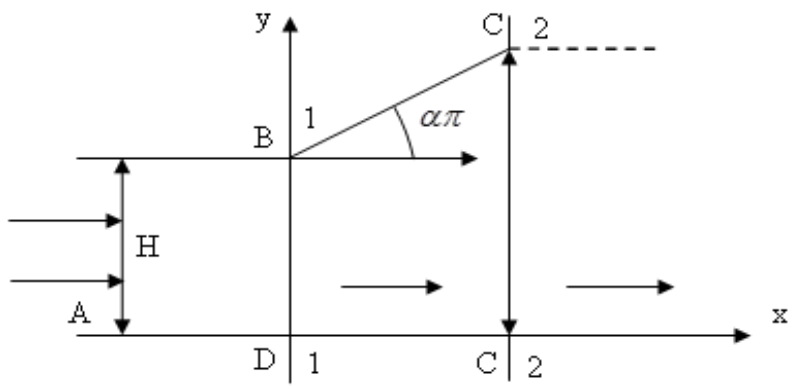

Fig.1. Symmetry of the flow area

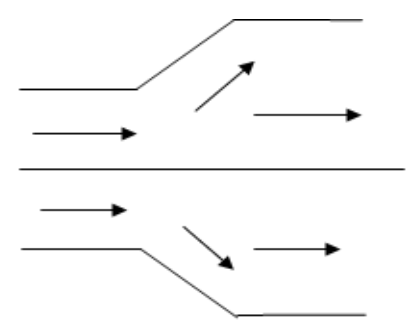

Fig.2. Flow area 
Due to the symmetric force of the flow area of the mixture of air and fibrous waste (Fig. 2), work was carried out on the pressure loss when passing through the section (CC), creating a turning zone that requires additional energy consumption. In this regard, it is not difficult to create a smooth contour that provides a smooth circulation of air and the flow of the mixture of fibrous waste on the flow curve [11, 12]. In this case, using Fig. 1 and 2 to solve the problem, we will create the equations of motion of a mixture of air and fibers in section 2-2 (Fig. 1) located in section $1-1$ and at a sufficient distance from it before the start of expansion of the air duct.

\subsection{Movement of a mixture of air and fibers}

Let us write the Bernoulli equation for the mixture for the cross-sections selected in this way during the movement of the mixture of air and fiber:

$$
P_{A}+\frac{1}{2}\left(\rho_{1} v_{1 A}^{2}+\rho_{2} v_{2 A}^{2}\right)=P_{C}+\frac{1}{2}\left(\rho_{1} v_{1 C}^{2}+\rho_{2} v_{2 C}^{2}\right)
$$

Here to reduce the pressure of the mixture.

$$
\Delta P=P_{A}-P_{C}=\frac{1}{2}\left[\left(\rho_{1} v_{1 C}^{2}+\rho_{2} v_{2 C}^{2}\right)-\left(\rho_{1} v_{1 A}^{2}+\rho_{2} v_{2 A}^{2}\right)\right]
$$

where $\rho_{1}$ and $\rho_{2}$ are the density of air and fibrous waste.

Applying the theory of the amount of motion affecting this mass and the change in the mass of a mixture of air and fibrous waste, we obtain the following formula.

$$
\left(P_{A}-P_{C}\right) H-x
$$

After calculating the volume associated with two sections of the wall, we write the volume of movement per unit time of the mass of the mixture of air and fibrous waste in the projection onto the deflecting wall BC:

$$
\left(\rho_{1} q_{1} v_{1 C}+\rho_{2} q_{2} v_{2 C}\right) \sin \alpha \pi-\left(\rho_{1} q_{1} v_{1 A}+\rho_{2} q_{2} v_{2 A}\right) \cos \alpha \pi
$$

Further, equation (2) is equated to the equation (3):

$$
\begin{aligned}
& \left(P_{A}-P_{C}\right) H-x=\left(\rho_{1} q_{1} v_{1 C}+\rho_{2} q_{2} v_{2 C}\right) \sin \alpha \pi-\left(\rho_{1} q_{1} v_{1 A}+\rho_{2} q_{2} v_{2 A}\right) \cos \alpha \pi \\
& x=\left(P_{A}-P_{C}\right) H-\left(\rho_{1} q_{1} v_{1 C}+\rho_{2} q_{2} v_{2 C}\right) \sin \alpha \pi+\left(\rho_{1} q_{1} v_{1 A}+\rho_{2} q_{2} v_{2 A}\right) \cos \alpha \pi
\end{aligned}
$$

Here, taking into account (1), we obtain the following formula:

$$
\begin{aligned}
& x=\frac{H}{2}\left[-\left(\rho_{1} v_{1 A}^{2}+\rho_{2} v_{2 A}^{2}\right)+\left(\rho_{1} v_{1 C}^{2}+\rho_{2} v_{2 C}^{2}\right)\right]-\left(\rho_{1} q_{1} v_{1 C}+\rho_{2} q_{2} v_{2 C}\right) \sin \alpha \pi+ \\
& +\left(\rho_{1} q_{1} v_{1 A}+\rho_{2} q_{2} v_{2 A}\right) \cos \alpha \pi
\end{aligned}
$$

In this case, the resistance coefficient for each pass (CC) in the direction is determined by M.I. Gurevich [11].

$$
C_{x}=\frac{x}{\frac{1}{2}\left(\rho_{1} v_{1 C}^{2}+\rho_{2} v_{2 C}^{2}\right) \cdot L}
$$

Then

$$
\begin{gathered}
C_{x}=\frac{2}{\left(\rho_{1} v_{1 C}^{2}+\rho_{2} v_{2 C}^{2}\right) \cdot L} \cdot\left\{\frac{H}{2}\left[\left(\rho_{1} v_{1 C}^{2}+\rho_{2} v_{2 C}^{2}\right)-\left(\rho_{1} v_{1 A}^{2}+\rho_{2} v_{2 A}^{2}\right)\right]-\right. \\
\left.-\left(\rho_{1} q_{1} v_{1 C}+\rho_{2} q_{2} v_{2 C}\right) \sin \alpha \pi+\left(\rho_{1} q_{1} v_{1 A}+\rho_{2} q_{2} v_{2 A}\right) \cos \alpha \pi\right\}= \\
=\frac{H}{L} \frac{\left(\rho_{1} v_{1 C}^{2}+\rho_{2} v_{2 C}^{2}\right)-\left(\rho_{1} v_{1 A}^{2}+\rho_{2} v_{2 A}^{2}\right)}{\rho_{1} v_{1 C}^{2}+\rho_{2} v_{2 C}^{2}}+2 \cdot \frac{\rho_{1} q_{1} v_{1 A}+\rho_{2} q_{2} v_{2 A}}{L \cdot\left(\rho_{1} v_{1 C}^{2}+\rho_{2} v_{2 C}^{2}\right)} \cos \alpha \pi- \\
-2 \cdot \frac{\rho_{1} q_{1} v_{1 C}+\rho_{2} q_{2} v_{2 C}}{L \cdot\left(\rho_{1} v_{1 C}^{2}+\rho_{2} v_{2 C}^{2}\right)} \sin \alpha \pi
\end{gathered}
$$

Considering the following

$$
q_{1}=H \cdot v_{1 A} \cdot f_{1}, \quad q_{2}=H \cdot v_{2 A} \cdot f_{2}
$$


Then

$$
\begin{aligned}
& C_{x}=\frac{H}{L}\left(1-\frac{\rho_{1} v_{1 A}^{2}+\rho_{2} v_{2 A}^{2}}{\rho_{1} v_{1 C}^{2}+\rho_{2} v_{2 C}^{2}}\right)+2 \cdot \frac{\rho_{1} \cdot H \cdot f_{1} \cdot v_{1 A}^{2}+\rho_{2} \cdot H \cdot f_{2} \cdot v_{2 A}^{2}}{L \cdot\left(\rho_{1} v_{1 C}^{2}+\rho_{2} v_{2 C}^{2}\right)} \cos \alpha \pi- \\
& -2 \cdot \frac{\rho_{1} \cdot H \cdot v_{1 A} \cdot f_{1} \cdot v_{1 C}+\rho_{2} \cdot H \cdot v_{2 A} \cdot f_{2} \cdot v_{2 C}}{L \cdot\left(\rho_{1} v_{1 C}^{2}+\rho_{2} v_{2 C}^{2}\right)} \sin \alpha \pi=\frac{H}{L}\left(1-\frac{\rho_{1} v_{1 A}^{2}\left(1+\frac{\rho_{2}}{\rho_{1}} \cdot \frac{v_{2 A}^{2}}{v_{1 A}^{2}}\right)}{\rho_{1} v_{1 C}^{2}\left(1+\frac{\rho_{2}}{\rho_{1}} \cdot \frac{v_{2 C}^{2}}{v_{1 C}^{2}}\right)}\right)+ \\
& +2 \cdot \frac{\rho_{1} \cdot H \cdot v_{1 A}^{2}\left(f_{1}+f_{2} \frac{\rho_{2}}{\rho_{1}} \cdot \frac{v_{2 A}^{2}}{v_{1 A}^{2}}\right)}{L \cdot \rho_{1} v_{1 C}^{2}\left(1+\frac{\rho_{2}}{\rho_{1}} \cdot \frac{v_{2 C}^{2}}{v_{1 C}^{2}}\right)} \cos \alpha \pi-2 \cdot \frac{\rho_{1} \cdot H \cdot v_{1 C}^{2}\left(f_{1} \cdot \frac{v_{1 A}}{v_{1 C}}+f_{2} \frac{\rho_{2}}{\rho_{1}} \cdot \frac{v_{2 A} \cdot v_{2 C}}{v_{1 C}^{2}}\right)}{L \cdot \rho_{1} v_{1 C}^{2}\left(1+\frac{\rho_{2}}{\rho_{1}} \cdot \frac{v_{2 C}^{2}}{v_{1 C}^{2}}\right)} \sin \alpha \pi \\
& C_{x}=\frac{H}{L}\left(1-\left(\frac{v_{1 A}}{v_{1 C}}\right)^{2} \frac{1+\frac{\rho_{2}}{\rho_{1}} \cdot\left(\frac{v_{2 A}}{v_{1 A}}\right)^{2}}{1+\frac{\rho_{2}}{\rho_{1}} \cdot\left(\frac{v_{2 C}}{v_{1 C}}\right)^{2}}\right)+2 \cdot \frac{H}{L}\left(\frac{v_{1 A}}{v_{1 C}}\right)^{2} \cdot \frac{f_{1}+f_{2} \frac{\rho_{2}}{\rho_{1}} \cdot\left(\frac{v_{2 A}}{v_{1 A}}\right)^{2}}{1+\frac{\rho_{2}}{\rho_{1}} \cdot\left(\frac{v_{2 C}}{v_{1 C}}\right)^{2}} \cos \alpha \pi- \\
& -2 \cdot \frac{H}{L} \cdot \frac{\left(f_{1} \cdot \frac{v_{1 A}}{v_{1 C}}+f_{2} \frac{\rho_{2}}{\rho_{1}} \cdot \frac{v_{2 A} \cdot v_{2 C}}{v_{1 C}^{2}}\right)}{1+\frac{\rho_{2}}{\rho_{1}} \cdot\left(\frac{v_{2 C}}{v_{1 C}}\right)^{2}} \sin \alpha \pi
\end{aligned}
$$

Into the resulting equations we introduce the following: $f(A C)=\frac{v_{1 A}}{v_{1 C}} ; \hat{\rho}_{2}=\frac{\rho_{2}}{\rho_{1}}, f(A)=\frac{v_{2 A}}{v_{1 A}} ; f(C)=\frac{v_{2 C}}{v_{1 C}}$, In this case

$$
\begin{gathered}
C_{x}=\frac{H}{L}\left(1-f^{2}(A C) \cdot \frac{1+\hat{\rho}_{2} \cdot f^{2}(A)}{1+\hat{\rho}_{2} \cdot f^{2}(C)}\right)+2 \cdot \frac{H}{L} f^{2}(A C) \cdot \frac{f_{1}+f_{2} \hat{\rho}_{2} \cdot f^{2}(A)}{1+\hat{\rho}_{2} \cdot f^{2}(C)} \cos \alpha \pi- \\
-2 \cdot \frac{H}{L} \cdot \frac{f_{1} \cdot f(A C)+f_{2} \cdot \hat{\rho}_{2} \cdot \frac{v_{2 A}}{v_{1 C}} \cdot f(C)}{1+\hat{\rho}_{2} \cdot f^{2}(C)} \sin \alpha \pi \\
C_{x}=\frac{1}{1+\hat{\rho}_{2} \cdot f^{2}(C)}\left\{\frac{H}{L}\left(1+\hat{\rho}_{2} \cdot f^{2}(C)-f^{2}(A C) \cdot\left(1+\hat{\rho}_{2} \cdot f^{2}(A)\right)\right)+\right. \\
+\left[2 \cdot \frac{H}{L} f^{2}(A C)\left(f_{1}+f_{2} \hat{\rho}_{2} \cdot f^{2}(A)\right) \cos \alpha \pi-\right. \\
\left.\left.-2 \cdot \frac{H}{L} \cdot\left(f_{1} \cdot f(A C)+f_{2} \cdot \hat{\rho}_{2} \cdot \frac{v_{2 A}}{v_{1 C}} \cdot f(C)\right) \sin \alpha \pi\right]\right\}
\end{gathered}
$$

Using the equality of the amounts of a mixture of air and fibrous waste in the inlet and outlet parts of the $q_{1}=q_{2}$ pipeline, we obtain the following equation.

$$
\left.\left.\begin{array}{c}
q_{1 A}=H f_{1} v_{1 A},-q_{1 A}=L f_{1} v_{1 C} \\
q_{2 A}=H f_{2} v_{2 A},-q_{2 A}=L f_{2} v_{2 C}
\end{array}\right\} \Rightarrow \begin{array}{l}
q_{1 A}=q_{1 C} \\
q_{2 A}=q_{2 C}
\end{array}\right\} \Rightarrow
$$


Taking into account the equality $v_{1 C}=\frac{H}{L} v_{1 A} ; v_{2 C}=\frac{H}{L} v_{2 A}$, we present the following equation:

$$
\left.\begin{array}{c}
f(C)=\frac{v_{2 C}}{v_{1 C}}=\frac{\frac{H}{L} v_{2 A}}{\frac{H}{L} v_{1 A}}=\frac{v_{2 A}}{v_{1 A}} \Rightarrow f(C)=f(A)=\frac{v_{2 A}}{v_{1 A}} \\
f(A C)=\frac{v_{1 A}}{v_{1 C}}=\frac{v_{1 A}}{\frac{H}{L} v_{1 A}}=\frac{L}{H}
\end{array}\right\}
$$

Taking into account equations (5) and (7) for the coefficient of local resistance, we obtain the following formula.

$$
\begin{gathered}
C_{x}=\frac{1}{1+\hat{\rho}_{2} f^{2}(A)}\left\{\left[\frac{H}{L}\left(1+\hat{\rho}_{2} f^{2}(A)\right)-\left(1+\hat{\rho}_{2} f^{2}(A) \frac{L}{H}\right)\right]+\right. \\
+2\left[\frac{H}{L}\left(\frac{L}{H}\right)^{2}\left(f_{1}+f_{2} \hat{\rho}_{2} f^{2}(A)\right) \cos \alpha \pi-\frac{H}{L}\left(f_{1} \cdot \frac{L}{H}+f_{2} \cdot \hat{\rho}_{2} \cdot \frac{v_{2 A}}{v_{1 C}} \cdot f(A)\right) \sin \alpha \pi\right] \\
C_{x}=\left(\frac{H}{L}-\frac{L}{H}\right)+2\left[\frac{L}{H}\left(f_{1}+f_{2} \hat{\rho}_{2} f^{2}(A)\right) \cos \alpha \pi-\right. \\
\left.-\frac{H}{L}\left(f_{1} \cdot \frac{L}{H}+f_{2} \cdot \hat{\rho}_{2} \cdot \frac{v_{2 A}}{v_{1 C}} \cdot f(A)\right) \sin \alpha \pi\right] /\left(1+\hat{\rho}_{2} f^{2}(A)\right)
\end{gathered}
$$

$C_{x}$ - local resistance, if the concentration of phases $f_{1}, f_{2}\left(f_{1}+f_{2}=1\right)$,

$\rho_{1}$ and $\rho_{2}$ - density of air and fibrous waste, $\mathrm{H}$ and $\mathrm{L}$ - if the width of the channel and the initial velocity of each area are known, then the coefficient of local resistance for each expanding pipeline is determined.

Here $0<\alpha<\frac{1}{2}$ that is $\left(0<\alpha \pi<\frac{\pi}{2}\right)$.

Thus, the main parameter, in addition to those listed above, is calculated according to the angle of the horizontal axis $\mathrm{OX}$, inclined to the upper wall $\alpha \pi$.

If we do not take into account the concentration of the $f_{1}, f_{2}$, phases in this case

$$
\begin{gathered}
C_{x}=\frac{H^{2}-L^{2}}{H L}+2\left[\frac{L}{H}\left(1+\hat{\rho}_{2} f^{2}(A)\right) \cos \alpha \pi-\frac{H}{L}\left(\frac{L}{H}+\hat{\rho}_{2} \cdot \frac{v_{2 A}}{v_{1 C}} \cdot f(A)\right) \sin \alpha \pi\right] \frac{1}{\left(1+\hat{\rho}_{2} f^{2}(A)\right)} \\
C_{x}=\frac{H^{2}-L^{2}}{H L}+2\left[\frac{L}{H} \cos \alpha \pi-\frac{H}{L} \cdot\left(\frac{L}{H}+\frac{v_{1 A}}{v_{1 C}}\right) \sin \alpha \pi\right]>0 \\
C_{x}=\frac{H^{2}-L^{2}}{H L}+2\left[\frac{L}{H} \cos \alpha \pi-\frac{H}{L} \cdot\left(\frac{L}{H}+f(A C)\right) \sin \alpha \pi\right]>0 \\
C_{x}=\frac{H^{2}-L^{2}}{H L}+2\left[\frac{L}{H} \cos \alpha \pi-\frac{H}{L} \cdot\left(\frac{L}{H}+\frac{L}{H}\right) \sin \alpha \pi\right]>0
\end{gathered}
$$

As a result, we get the following formula:

$$
C_{x}=\frac{H^{2}-L^{2}}{H L}+2\left[\frac{L}{H} \cos \alpha \pi-2 \sin \alpha \pi\right]>0
$$

\section{Analytical calculation of theoretical research}

From the obtained equations, taking into account the diameter of the inlet air duct $H=0,2$, and the diameter of the outlet air duct $L=0,4$, the effect of the expanding pipeline on the local resistance of the expanding angle was studied (Fig. 3). 


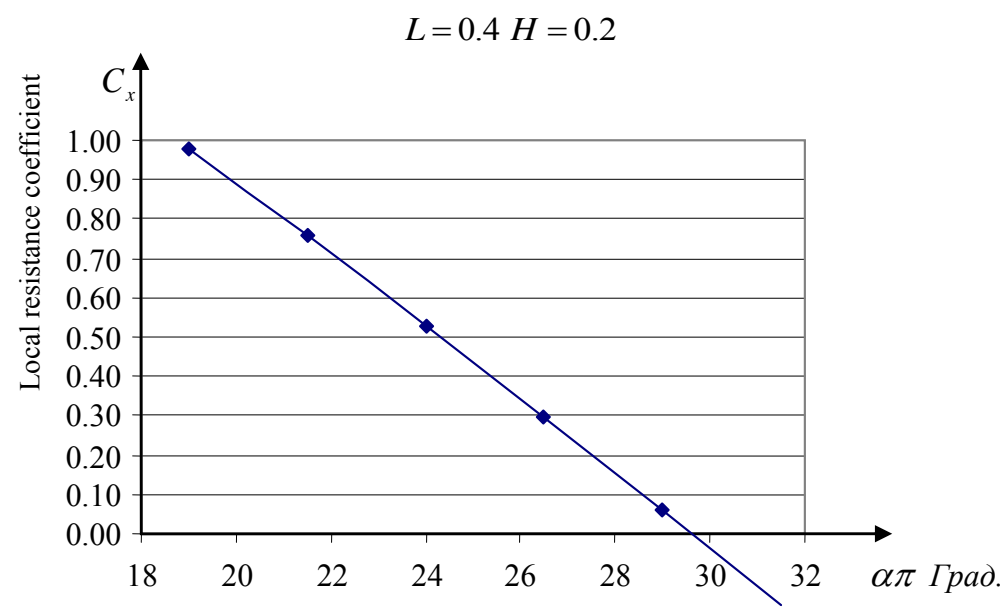

Expansion angle of the expanding pipeline

Fig.3. A graph of the relationship between the expansion angle of the expanding pipeline $\alpha \pi$ and the coefficient of local resistance $C_{x}$

Fig. 3 we can see that if the optimal value of the local resistance is $\alpha \pi=29^{\circ}$, then the local resistance will be equal to $C_{x} \approx 0,06$.

Based on the obtained equations, we will conduct theoretical studies on the ratio of the velocities of fibrous waste in incoming and outgoing pipelines.

$$
\begin{gathered}
\frac{v_{1 A}}{v_{2 A}}=\frac{v_{1 C}}{v_{2 C}}, \frac{v_{1 C}}{v_{1 A}}=\frac{v_{2 C}}{v_{2 A}} \\
\frac{H}{L}=\frac{v_{1 C}}{v_{1 A}}=\frac{v_{2 C}}{v_{2 A}}, \frac{L}{H}=\frac{v_{1 A}}{v_{1 C}}=\frac{v_{2 A}}{v_{2 C}} \\
C_{x}=\frac{H^{2}-L^{2}}{H L}+2\left[\frac{L}{H} \cos \alpha \pi-2 \sin \alpha \pi\right]>0 \\
C_{x}=\left(\frac{H}{L}-\frac{L}{H}\right)+2\left[\frac{L}{H} \cos \alpha \pi-2 \sin \alpha \pi\right]>0 \\
C_{x}=\left(\frac{v_{2 C}}{v_{2 A}}-\frac{L}{H}\right)+2\left[\frac{L}{H} \cos \alpha \pi-2 \sin \alpha \pi\right]>0 \\
\frac{v_{2 C}}{v_{2 A}}=C_{x}+\frac{L}{H}+2\left[\frac{L}{H} \cos \alpha \pi-2 \sin \alpha \pi\right]>0 \\
v_{2 C}=C_{x} v_{2 A}+\frac{L}{H} v_{2 A}+2 v_{2 A}\left[\frac{L}{H} \cos \alpha \pi-2 \sin \alpha \pi\right]>0
\end{gathered}
$$

Let us find the speed of the outgoing fibrous waste, with the known optimal values of the local resistance, which is equal to the local resistance equal to $C_{x} \approx 0.06$. Here, we arbitrarily choose the diameter of the incoming pipeline $\mathrm{H}=$ $0.2 \mathrm{~m}$, the diameter of the outgoing pipeline $\mathrm{L}=0.4 \mathrm{~m}$, as well as the speed of the incoming fibrous waste (Fig. 4). Drawing conclusions from the graph obtained from Fig. 4, in conclusion, we can say that the theoretical studies carried out were carried out correctly taking into account the particle velocities, we can see that the velocities of the fibrous waste at the entrance and exit are reduced by 2 times. As a result, when developing devices for capturing fibrous waste, if we take into account that the angle of expansion during expansion of pipes is equal to $\alpha \pi=29^{\circ}$, then local resistances become less. In addition, taking into account that $\frac{v_{2 C}}{v_{2 A}}=0.5$, then the speed of fibrous waste in the chamber is reduced by an average of 2 times. This will help to find theoretical solutions for the retention of pipes for pneumatic conveying of fibrous waste in our subsequent studies. 


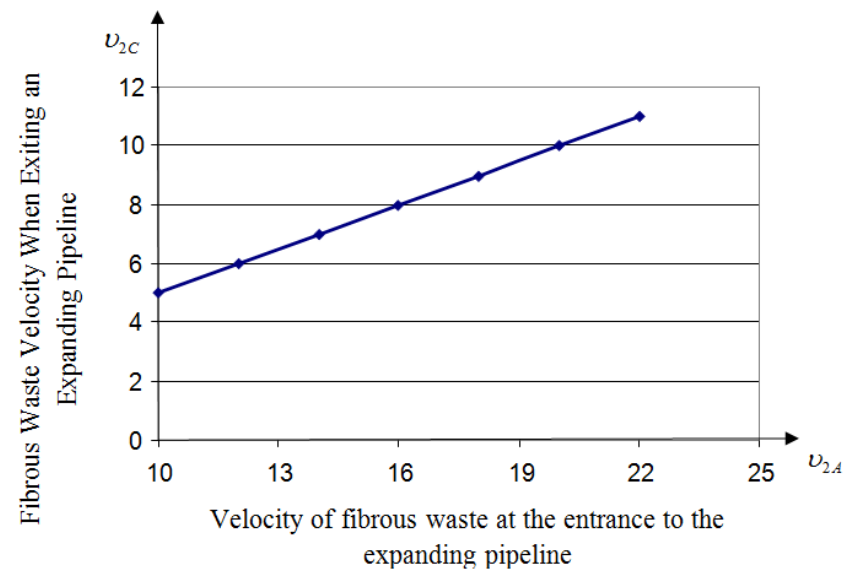

Fig.4. The relationship of the velocities of incoming and outgoing fibrous waste in the expanding part of the pipeline

\section{Conclusions}

According to the results obtained, it was proved that the local resistance is equal to the value, at the optimal values $C_{x} \approx 0.06$ of the expansion angle of the expanding pipeline of local resistance equal to $\alpha \pi=29^{\circ}$.

In addition, theoretical equations have been developed, with the diameter of the outgoing pipeline equal and with the diameter of the pipeline the velocity of the mixture of air and fibrous waste, the optimal values $H=0.2$ of local resistance equal to $L=0,4$. The analyzes carried out show that the flow through the local resistance of the air or mixture constantly comes on a par with the pressure increases. Due to the fact that the analytical developments and theoretical studies carried out on this topic have not been widely studied, equations were developed for calculating the local resistance of a mixture of air and fibrous waste in expanding pipelines and was proved by analyzing the particle velocities.

The value of local resistance is theoretically determined, which is equal at the optimal value of the mixture of air and fibrous waste in the expanding pipeline, which is equal to $\alpha \pi=29^{\circ}$. In order to prove the accuracy of the calculation of particle velocities, according to the studies, the velocities of fibrous waste at the entrance and exit from the pipeline were studied. As a result, when developing devices for capturing fibrous waste, if we take into account that the angle of expansion during expansion of pipes is equal to $\alpha \pi=29^{\circ}$, then local resistances become less. In addition, taking into account that $\frac{v_{2 C}}{v_{2 A}}=0.5$, then the speed of fibrous waste in the chamber is reduced by an average of 2 times. This will help to find theoretical solutions for the retention of pipes for pneumatic conveying of fibrous waste in our subsequent studies.

\section{References}

1. L. Wang, C. B. Parnell, B. W. Shaw, Performance Characteristics of Cyclones in Cotton-Gin Dust Removal, Agricultural Engineering International: the CIGR Journal of Scientific Research and Development 6, 02001 (2002)

2. M. Khodjiev, I. Abbazov, I. Makhkamov, J. Karimov, M. Rajabova, Technological breakthrough of cotton cleaning enterprises fiber waste, Solid State Technology 63, 490 (2020)

3. K. Muksin, A. Ilkhom, K. Javlon, A New Technology for Dust Removal From Cotton Processing, Int. J. Recent Technology and Engineering 8, 583-596 (2019)

4. V. Kuznetsov, S. Denisov, Pneumotransport of woodworking enterprises, Bratsk State Technical University, Bratsk (2007)

5. K. Hoganson, D. Gross, Patent USA, Variable speed drive for pneumatic transport system, US 7,950,879 B2. 05/31/2011.

6. P. V. Baydyuk, M. Kh. Khozhiev, Methodology for determining hydraulic pressure losses in a horizontal working pipeline of a pneumatic conveying system for raw cotton, Cotton Industry 2, 8-9 (1992)

7. I. Abbazov, M. Khodjiyev, O. Alimov, J. Karimov, Fraction structure of cotton cleaning equipment in cotton enterprises and their cleaning effectiveness, Int. J. Advanced Research in Science, Engineering and Technology 6, 7983-7988 (2019)

8. I. Z. Abbazov, M. T. Khodjiyev, Kh. S. Usmanov, Issues of purification of dusted air at cotton cleaning plants, Fundamental and Applied Research in the Modern World 21, 45-47 (2018) 
9. M. T. Khodjiev, O. N. Alimov, I. Z. Abbazov, Ways to effectively treat waste from ginneries, Textile Problems 1, 10-15 (2018)

10. M. T. Khodjiyev, I. Z. Abbazov, Problems of air purification of dust from ginneries, Problems of Mechanics 3-4, 145-148 (2013)

11. H. R. Vallentine, Flow of an Ideal Fluid, In: Applied Hydrodynamics, Springer, Boston, MA (1967)

12. R. F. Huang, S. Y. Lin, S. Y. Jan, R. H. Hsieh, Y. K. Chen, C. W. Chen, W. Y. Yeh, C. P. Chang, T. S. Shih, C. C. Chen, Aerodynamic characteristics and design guidelines of push-pull ventilation systems, Ann Occup Hyg. 49(1), 1-15 (2005) 